\title{
Bortezomib induces apoptosis and suppresses cell growth and metastasis by inactivation of Stat3 signaling in chondrosarcoma
}

\author{
XING BAO ${ }^{1,2}$, TINGTING REN ${ }^{1,2}$, YI HUANG ${ }^{1,2}$, CHONGMIN REN $^{1,2}$, \\ $\mathrm{KANG}_{\mathrm{YANG}^{1,2}}$, HONGLIANG ZHANG ${ }^{1,2}$ and WEI GUO ${ }^{1,2}$ \\ ${ }^{1}$ Musculoskeletal Tumor Center, Peking University People's Hospital, Beijing 100044; \\ ${ }^{2}$ Beijing Key Laboratory of Musculoskeletal Tumor, Beijing 100044, P.R. China
}

Received August 17, 2016; Accepted October 7, 2016

DOI: 10.3892/ijo.2016.3806

\begin{abstract}
Bortezomib, formerly known as PS341, is a novel proteasome inhibitor with in vitro and in vivo antineoplastic effects in many malignancies. However, diverse antitumor mechanisms of bortezomib have been identified in many investigations and preclinical studies. Understanding the molecular and cellular mechanisms through which bortezomib acts will improve the therapeutic utility of this drug in different cancer types. In the present study, we investigated the in vitro and in vivo effects of bortezomib on chondrosarcoma. Bortezomib selectively inhibited cell growth in chondrosarcoma cells but not in normal articular cartilage cells. In addition to growth inhibition, apoptosis and cell cycle arrest, bortezomib triggered alleviation of migratory and invasive properties of chondrosarcoma cells. Mechanistically, signal transducer and activator of transcription 3 (Stat3) and its downstream targets $\mathrm{Bcl}-2$, cyclin D1 and c-Myc was inactivated by bortezomib treatment. Accordingly, small interfering RNA (siRNA)mediated Stat 3 knockdown enhanced bortezomib-induced apoptosis, and concomitantly enhanced the inhibitory effect of bortezomib on cell viability, migration and invasion. Moreover, while Slug, MMP9, MMP2, CD44, N-cadherin and vimentin, the mesenchymal cell markers, were repressed by bortezomib concomitant increased expression of E-cadherin was observed. In vivo, bortezomib downregulated Stat3 activity and mesenchymal cell marker expression, induced apoptosis and inhibition of metastasis and tumor growth. Together, inactivation of Stat 3 signaling contributes to bortezomibinduced inhibition of tumor growth, migration and invation on
\end{abstract}

Correspondence to: Professor Wei Guo, Musculoskeletal Tumor Center, Peking University People's Hospital, No. 11 Xizhimen South Street, Beijing 100044, P.R. China

E-mail: bonetumor@163.com

Abbreviations: Stat 3 , the transcription factor signal transducer and activator of transcription 3; IHC, immunohistochemistry; MET, mesenchymal-epithelial transition; siRNA, small interfering RNA; CCK-8, Cell Counting kit-8

Key words: chondrosarcoma, bortezomib, apoptosis, metastasis, MET, Stat3 signaling chondrosarcoma. Bortezomib demonstrates an antineoplastic role on chondrosarcoma both in vitro and in vivo. These beneficial effects can be explained by bortezomib-mediated Stat 3 supression. The present study suggests a promising therapeutics target in chondrosarcoma and probably in other kinds of metastatic malignant tumors.

\section{Introduction}

Chondrosarcoma is the second most commonly occurring primary bone tumor (1-3). At present, wide-margin surgical resection remains the main curative method of treatment for chondrosarcoma, which continues to have poor prognosis due to resistance to conventional chemo- and radiotherapy (4-6). Consequently, there exists a critical clinical need to explore novel molecular-targeted therapies for this disease.

The transcription factor signal transducer and activator of transcription 3 (Stat3) is a point of convergence for numerous oncogenic and inflammatory signaling pathways, including cytokines, growth factors and oncogenes (7-9). Stat 3 participates in cell growth and survival regulating cell proliferation, apoptosis and autophagy $(9,10)$. Constitutive activation of Stat3 represents a key player in tumor angiogenesis and metastasis and has been observed in other cancers of the bone including osteosarcoma (10-15). As such, Stat3 has emerged as a promising molecular target for cancer therapy.

Bortezomib, formerly known as PS341, is a selective proteasome inhibitor, which has been approved by the US Food and Drug Administration for the treatment of patients with multiple myeloma and mantle cell lymphoma $(16,17)$. Recently it was indicated that bortezomib also has crucial antineoplastic activity against some solid tumors, including ovarian and prostate cancers as well as squamous cell cancer of the head and neck, Ewing's sarcoma and osteosarcoma. Preclinical data have suggested that bortezomib's antineoplastic effect occurs via diverse mechanisms, exerting significantly different roles in a tissue and cancer-specific context (18-21). Moreover, it has been shown that inactivation of Stat 3 plays an important role in bortezomib-mediated treatment of myeloma (22). However, the effects of bortezomib on human chondrosarcoma have not been adequately studied and, in particular, the mechanisms by which bortezomib exerts its antitumor functions in human chondrosarcoma have not been previously investigated. 
While the majority of published studies on the antineoplastic actions of bortezomib have focused on suppression of proliferation and promotion of apoptosis $(17,19)$, little is known about the effects of bortezomib on modulating the cell cycle progression and metastasis in cancer.

In the present study, we investigated the in vitro and in vivo effects of bortezomib on human chondrosarcoma. In particular, we demonstrated the importance of Stat 3 signaling for the antitumor actions of this agent, including apoptosis promotion, cell cycle arrest, inhibition of migration and invasion. Thus, we report on the therapeutic potential of bortezomib on human chondrosarcoma, based on our improved understanding of this agent.

\section{Materials and methods}

Animals and ethical statement. Six-week-old BALB/c female athymic nude mice were obtained (Vital River Laboratories Co., Ltd., Beijing, China). The present study was carried out in accordance with the recommendations in the Guide for the Chinese Ethics Review Committees. The protocol was approved by the Ethics Committee of Peking University People's Hospital. All experiments was carried out under the ethics approval of Peking University People's Hospital and was conducted according to the National Institutes of Health guidelines. Nude mice were maintained under specific pathogen-free (SPF) conditions, and bedding, water and daily rations were sterilized.

Tissue specimen. The paraffin-embedded pathological specimens from 12 patients with chondrosarcoma and adjacent non-tumor tissues were obtained from the Department of Pathology and the Musculoskeletal Tumor Center, Peking University Peoples Hospital (Beijing, China). Informed consents (written in the light of the ethical guidelines) were obtained from all the patients. All human specimens were approved by the Research Ethics Committee of Peking University Peoples Hospital (Beijing, China).

Cell culture, cell viability assay and colony formation assay. The human articular chondrocyte cell line HC-a (Sciencell Research Laboratories, Carlsbad, CA, USA) was maintained in Dulbecco's modified Eagle's medium (DMEM) supplemented with $15 \%$ fetal bovine serum (FBS), plus antibiotics. SW1353 cells were obtained from the American Type Culture Collection (ATCC; Manassas, VA, USA) and were maintained in L-15 medium (Gibco, Grand Island, NY, USA). OUMS-27 cells, HCS-2/8 cells and JJ012 cells were kindly gifted from Dr J. Block (Rush Medical College, Chicago, IL, USA) and were cultured in DMEM (HyClone Laboratories, Inc., Logan, UT, USA) supplemented with $10 \%$ fetal calf serum (FCS; Gibco) at $37^{\circ} \mathrm{C}$ in a humidified atmosphere with $5 \% \mathrm{CO}_{2}$. All experiments were performed during the exponential phase of cell growth. Cell viability was assessed using the CCK- 8 assay as previously described (23). Cells were plated in 96-well plates at a density of 5,000 cells in $100 \mu 1$ medium/well one day before the experiment. The cells were treated with bortizomib (Sigma-Aldrich Chemical, Co., St. Louis, MO, USA) with indicated condition, which included gene knockdown. The cell viability was examined by CCK- 8 assay. For colony formation assay, following treatment, adherent cells were trypsinized and 1,000 viable cells were subcultured in 6-well plates (in triplicate). Cells were allowed to adhere and colonize for 14 days. Media were removed and cells were fixed in methyl alcohol for $15 \mathrm{~min}$ and stained with crystal violet staining solution for visualizing colonies, as previously described (23).

Western blot analysis. Equal amounts of proteins collected from cell lysates were loaded on 10-15\% SDS-PAGE gels using a NuPAGE system (Invitrogen, Carlsbad, CA, USA) and then transferred onto PVDF membranes as previously described (24). The following antibodies were used in the experiments: anti-p-STAT3, anti-STAT3, anti-c-Myc, anti-Ecadherin, anti-vimentin, anti-cyclin D1, anti-Bax, anti-Bcl-2, and anti-GAPDH were all from Cell Signaling Technology (Beverly, MA, USA). Anti-N-cadherin, anti-Slug, anti-MMP9 and anti-CD44 were from Abcam (Cambridge, MA, USA).

Immunohistochemistry. Paraffin sections were reacted with rabbit polyclonal anti-p-Stat3, anti-vimentin, anti-N-cadherin and anti-E-cadherin antibodies (1:200 dilution). Sections stained with non-immune rabbit serum (1:200 dilution) in phosphate-buffered saline (PBS) instead of primary antibody served as negative controls. Cells exhibiting positive staining on cell membranes and in the cytoplasm and nucleus were counted in at least 10 representative fields (x400 magnification) and the mean percentage of positive cells was calculated. Immunostaining was assessed by two independent pathologists blinded to clinical characteristics and outcomes.

Cell cycle and apoptosis analysis by flow cytometry. Cells for cell cycle analysis were fixed in $70 \%$ ethanol, digested with RNaseA and labeled with propidium iodide (PI). Apoptotic cells were analyzed with Annexin V/FITC kit (BD Biosciences, San Jose, CA, USA) according to the manufacturer's instructions and were analyzed by flow cytometry after compound treatment as previously described (25).

Wound healing assay. Cells ( $\left.2 \times 10^{5}\right)$ OUMS-27, HCS-2/8 and SW1353 were seeded into a 24-well plate. The tumor cells grew to confluence $24 \mathrm{~h}$ later. An artificial wound was introduced with a P10 pipette tip/well. Data of the wounded area were taken at 0 and $24 \mathrm{~h}$ with a microscope (Olympus Corp). The assay was repeated three times.

Transwell assay. HCS-2/8, OUMS-27 and SW1353 cells were respectively harvested, washed and suspended with DMEM (Gibco/Life Technologies, 11965-092), L-15 medium (Gibco) and seeded to the upper chambers of Transwell inserts ( $8 \mu \mathrm{m}$ pore size; Corning Incorporated, Corning, $\mathrm{NY}$, USA) with/without low concentration bortezomib $(10 \mathrm{nM})$ in the migration assay. The upper chambers were coated with Matrigel (BD Biosciences, 354234) prior to the inoculation of the cancer cells and bortezomib in the invasion assay. The lower compartments were filled with DMEM or L-15 medium supplemented with 5\% FBS. The cells in the upper chamber were removed with a swab after incubation for $12 \mathrm{~h}$ in the migration assay or $24 \mathrm{~h}$ in the invasion assay. The cells that migrated to the lower layer and attached to the membrane were stained with crystal violet and were numbered in five 
A

A

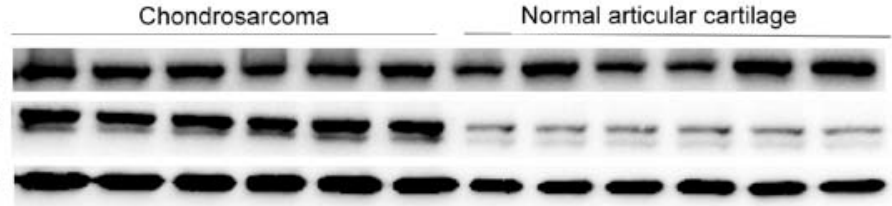

GAPDH

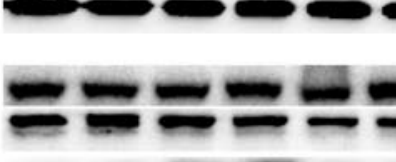

GAPDH

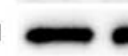

B
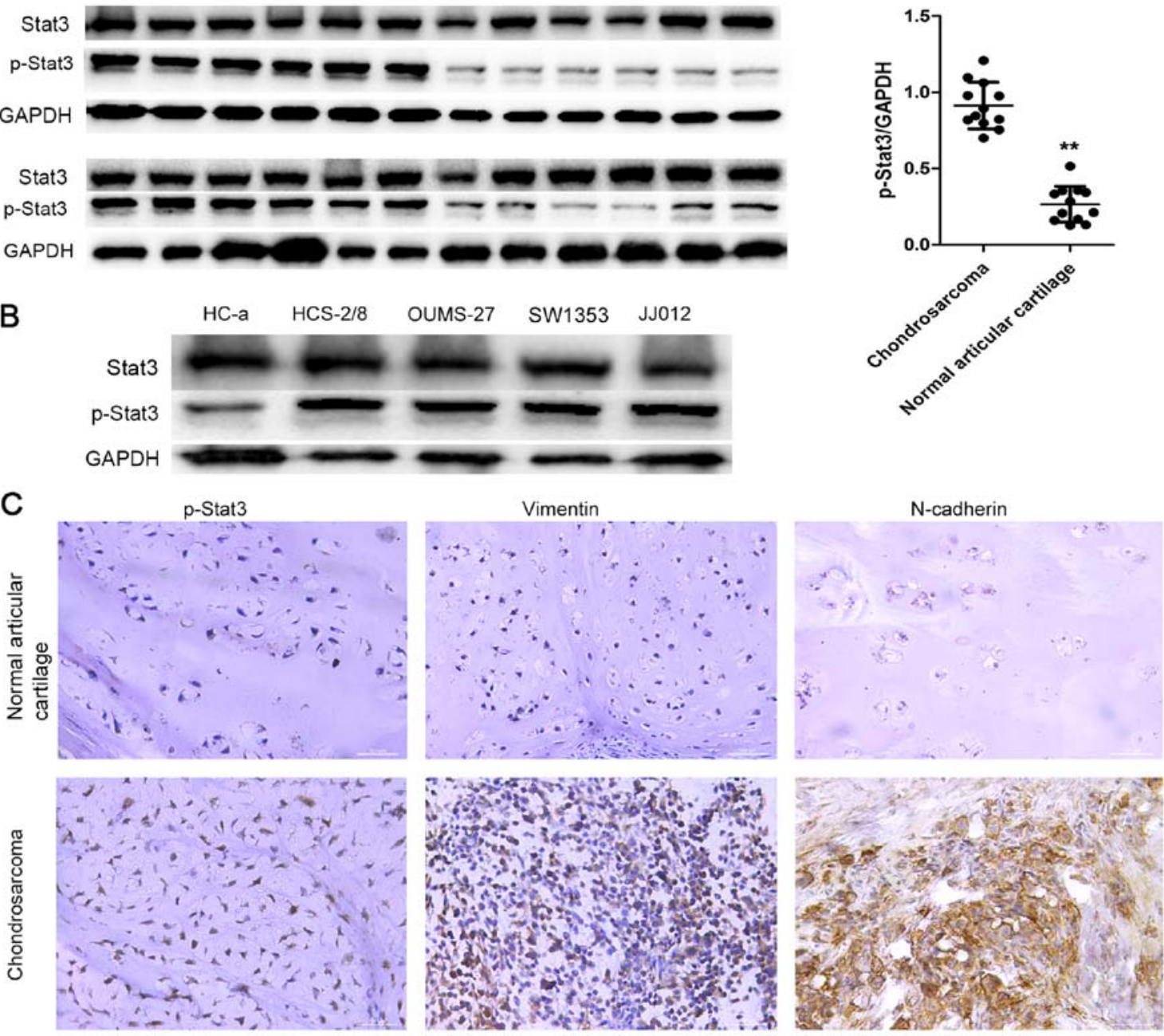

Figure 1. Expression of p-Stat3, vimentin and N-cadherin in normal articular cartilage, in chondrosarcoma and in their corresponding cell lines HC-a, HCS-2/8, OUMS-27, SW1353 and JJ012. (A) Western blot analysis demonstrates that p-Stat 3 was highly expressed in chondrosarcomas but low in the normal articular cartilage tissues (left panel). Average expression levels of p-Stat3 of normal articular cartilage and chondrosarcoma tissues (right panel). Data are presented as mean $\pm \mathrm{SD}(\mathrm{n}=12) .{ }^{* *} \mathrm{P}<0.05$. (B) Proteins were examined by western blotting in human articular chondrocyte cell line HC-a and chondrosarcoma cell lines HCS-2/8, OUMS-27, SW1353 and JJ012. (C) The expression of p-Stat3, vimentin and N-cadherin a cohort of 42 human chondrosarcoma specimens was determined by IHC staining. Representative images of p-Stat3, vimentin and $\mathrm{N}$-cadherin normal and chondrosarcoma specimens are shown.

fields per well under a microscope. The assay was repeated three times.

Mesenchymal-epithelial transition (MET) induction. OUMS-27 and HCS-2/8 cells were cultured as adherent cells in the complete medium overnight. The cells were maintained in either medium alone or medium supplemented with $2.5 \mathrm{ng} / \mathrm{ml}$ TGF- $\beta 1$ (R\&D Systems, Minneapolis, MN, USA), with or without $20 \mathrm{nM}$ bortezomib in a humidified $5 \% \mathrm{CO}_{2}$ incubator at $37^{\circ} \mathrm{C}$. The morphological images of the cells were taken seven days after the incubation. The experiments were repeated three times.

Gene knockdown using siRNA. The siRNAs to Stat 3 or control siRNA were all purchased from Suzhou GenePharma, Co., Ltd. (Suzhou, China). Cells were transfected with siRNA using Lipofectamine 3000, purchased from Origene Technologies Inc. (Rockville, MD, USA) according to the manufacturer's instructions. Cells were incubated for $48 \mathrm{~h}$ before further treatment.
Generation of xenografts. Six-week-old BALB/c female athymic nude mice (Vital River Laboratories) were subcutaneously injected in the right flank with cells $\left(2 \times 10^{6}\right.$ in $0.1 \mathrm{ml}$ PBS). Once a palpable tumor developed, the mice were randomly divided into two groups and intraperitoneally administered dimethyl sulfoxide (DMSO) or bortezomib at a dose of $0.5 \mathrm{mg} / \mathrm{kg}$ every other day for 30 days. The volume of xenografts was measured every 5 days (tumor volume $\left.\left.=(\text { length } \mathrm{x} \text { width})^{2}\right) / 2\right)$. The mice were sacrificed after 30 days. The tumor samples were processed for routine IHC. The tumor metastatic ability of HCS-2/8 cells $\left(5 \times 10^{6}\right)$ was observed following cell injection intravenously into the tail vein. Four weeks later, the mice were randomly divided into two groups and intraperitoneally administered with DMSO or bortezomib at a dose of $0.5 \mathrm{mg} / \mathrm{kg}$ every other day for 30 days ( $n=6$ per group), the mice were sacrificed and the number of metastatic nodules on the lung surface was counted. Metastatic lungs were fixed with $4 \%$ paraformaldehyde before dehydration and paraffin embedding. Paraffin sections were stained with hematoxylin and eosin according to the standard protocols. 
A
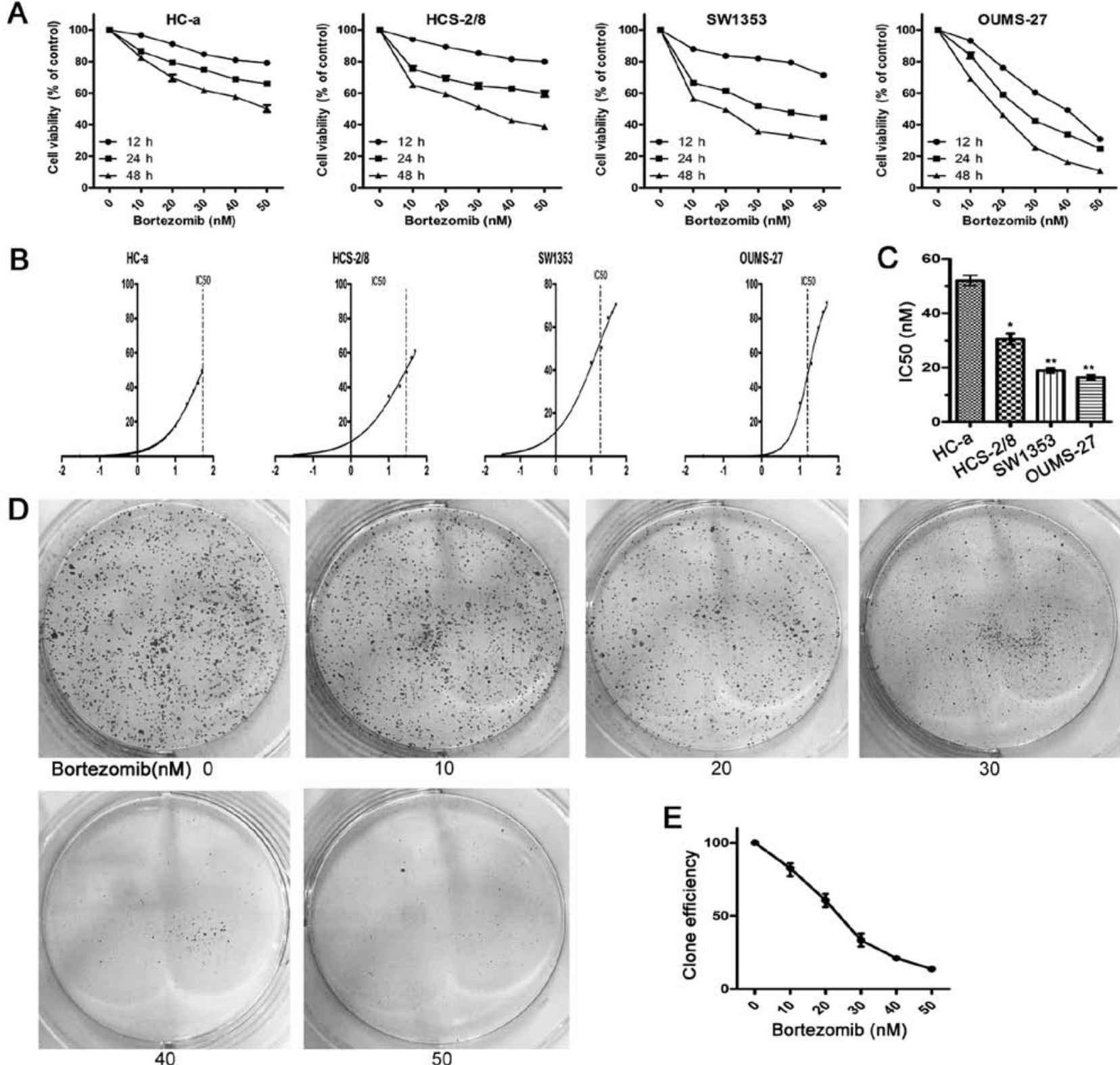

Figure 2. Bortezomib specifically inhibits the growth and promotes apoptosis of human chondrosarcoma cells. (A) Chondrosarcoma cells were treated with bortezomib at the indicated concentration for 12,24 and $48 \mathrm{~h}$. Cell viability, assayed by CCK- 8 , is presented as the means \pm SD from three separate experiments. (B) The vertical dashed line indicate the values of $\mathrm{IC}_{50}$ of bortezomib in HC-a, HCS-2/8, SW1353 and OUMS-27 cells. (C) The IC $\mathrm{C}_{50}$ of bortezomib in HC-a cells was significantly higher than that in HCS-2/8, OUMS-27 and SW1353. ${ }^{*} \mathrm{P}<0.05$ and ${ }^{* *} \mathrm{P}<0.01$ represent significant differences compared with the HC-a. (D and E) Colony formation of SW1353 cells was decreased in a dose-dependent manner by bortezomib treatment. Experiments were performed in triplicate.

Statistical analysis. Kaplan-Meier analysis was used to estimate the cumulative cause-specific survival rate and the differences in mouse survival with DMSO/bortezomib ( $\mathrm{n}=5$ per group). The influence of bortezomib on the growth, apoptosis, migration, invation, cell cycle and tumor formation of chondrosarcoma cells were analyzed by the Student's t-test. In all statistical analyses, statistical significance in the two-sided test was indicated with $\mathrm{P} \leq 0.05$ and $\mathrm{P}<0.01$ was remarkably significant.

\section{Results}

p-Stat 3 and EMT-related protein expression in the normal articular cartilage, in chondrosarcoma and in their corre- sponding cell lines HC-a, OUMS-27, HCS-2/8, SW1353 and $J J 012$. We examined the expression of p-Stat 3 using western blot analysis in 12 samples taken from the normal articular cartilage and chondrosarcoma tissues, respectively. Western blot analysis showed that p-Stat 3 was highly expressed in chondrosarcomas and considerably lower in the normal articular cartilage tissues (Fig. 1A and B). These results indicated that p-Stat 3 might have tumor-promoting roles in chondrosarcoma. To validate our results, we analyzed the expression of p-Stat3 in the corresponding cell lines HC-a, HCS-2/8, OUMS-27, SW1353 and JJ012 by western blotting and observed similar results (Fig. 1C).

We also assessed the expression of p-Stat 3 and vimentin with immunohistochemistry (IHC). Weak expression of 

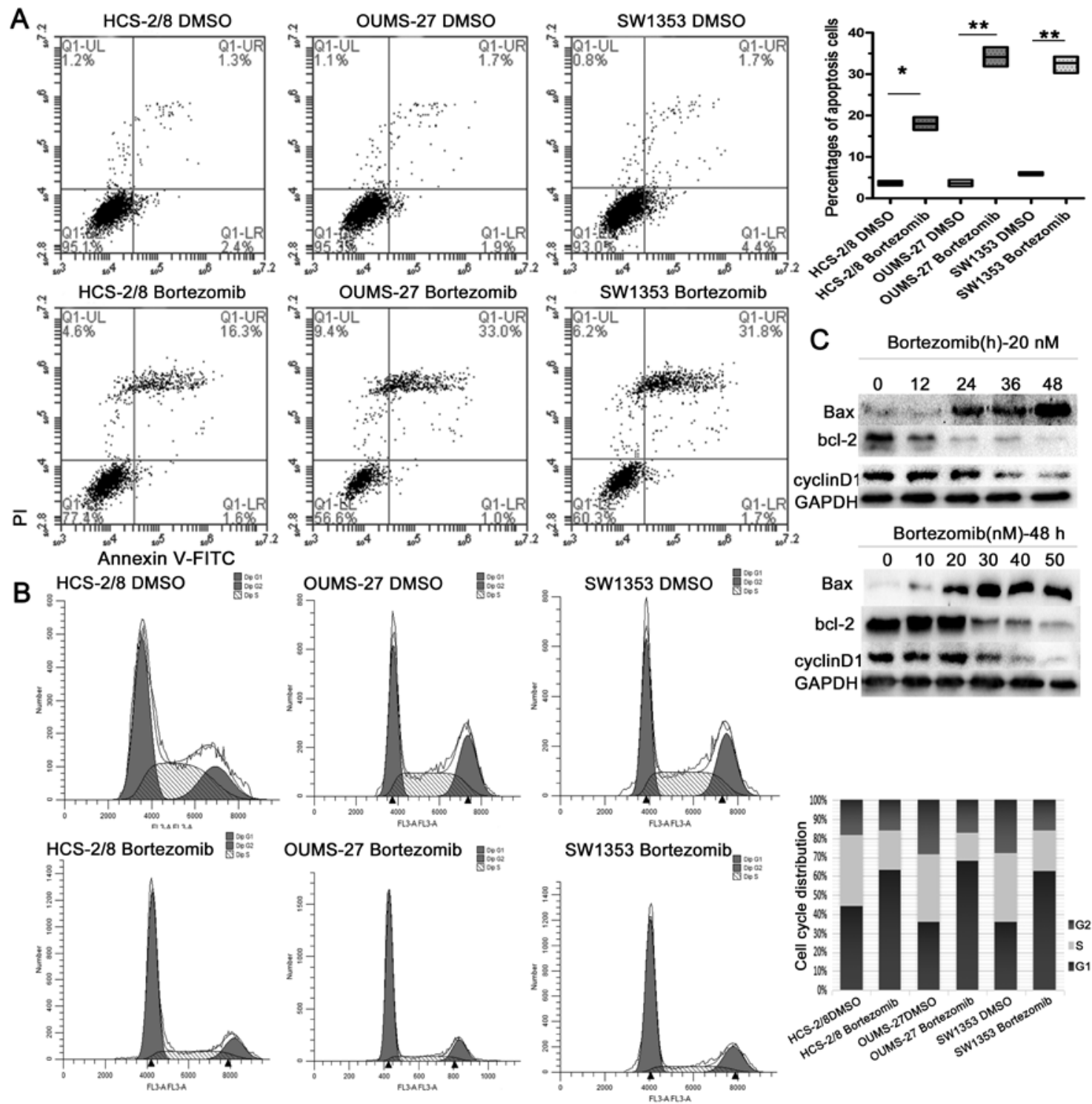

Figure 3. Bortezomib induces chondrosarcoma apoptotic cell death and promotes G0/G1 phase arrest. (A) Bortezomib induces apoptosis of chondrosarcoma cells. The apoptosis cells were detected with Annexin V-FITC and PI staining. (B) Bortezomib induces G0/G1 cell cycle arrest in chondrosarcoma cells DMSO or bortezomib was supplemented into the medium of HCS-2/8, OUMS-27 and SW1353 cells and cell cycle analysis was processed after $70 \%$ ethanol fixation and PI staining. (C) Effects of bortezomib on apoptosis and G0/G1 phase-related proteins assayed by western blot analysis at different treatment concentrations and different times. Protein was resolved by SDS-PAGE with GAPDH as a control $\left({ }^{*} \mathrm{P}<0.05,{ }^{* *} \mathrm{P}<0.01\right)$.

p-Stat3, N-cadherin and vimentin was observed in the normal articular cartilage, but strong expression was recorded in the chondrosarcoma tissues (Fig. 1D).

Bortezomib specifically inhibits the growth and promotes apoptosis of human chondrosarcoma cells. To investigate the effect of bortezomib on growth of human chondrosarcoma cells, we first examined the function of bortezomib in three human chondrosarcoma cell lines (OUMS-27, HCS-2/8 and SW1353) and human articular chondrocyte cells (HC-a), respectively. We cultured each cell line with different concentration of bortezomib for 12, 24 or $48 \mathrm{~h}$ and analyzed cell viability. The growth of human chondrosarcoma cells was inhibited by bortezomib in a dose- and time-dependent manner. By contrast, the viability of HC-a cells only decreased slightly (Fig. 2A). As shown in Fig. 2B and C, the $\mathrm{IC}_{50}$ of bortezomib in $\mathrm{HC}$-a cells was higher than that needed for chondrosarcoma cells (HCS-2/8, OUMS-27 and SW1353). In colony formation assays, bortezomib decreased colony formation compared with control (Fig. 2D and E). These data showed that bortezomib specifically suppressed the growth of human chondrosarcoma cells but not normal cells in vitro.

Bortezomib induces apoptotic cell death and promotes G0/GI phase arrest. To investigate the role of bortezomib in cell death of chondrosarcoma cells, chondrosarcoma cells were analyzed by flow cytometry following Annexin V-FITC and propidium iodide (PI) dual staining. As shown in Fig. 3A, bortezomib significantly induced apoptosis. As key executors of cell apoptosis, the ratio of Bax to Bcl-2 proteins increased following a 48-h treatment with bortezomib, or with $20 \mathrm{nM}$ bortezomib for indicated time-points (Fig. 3C).

As previously reported, siRNA-mediated inhibition of STAT3 promoted G0/G1 cell cycle arrest (26). We evaluated 

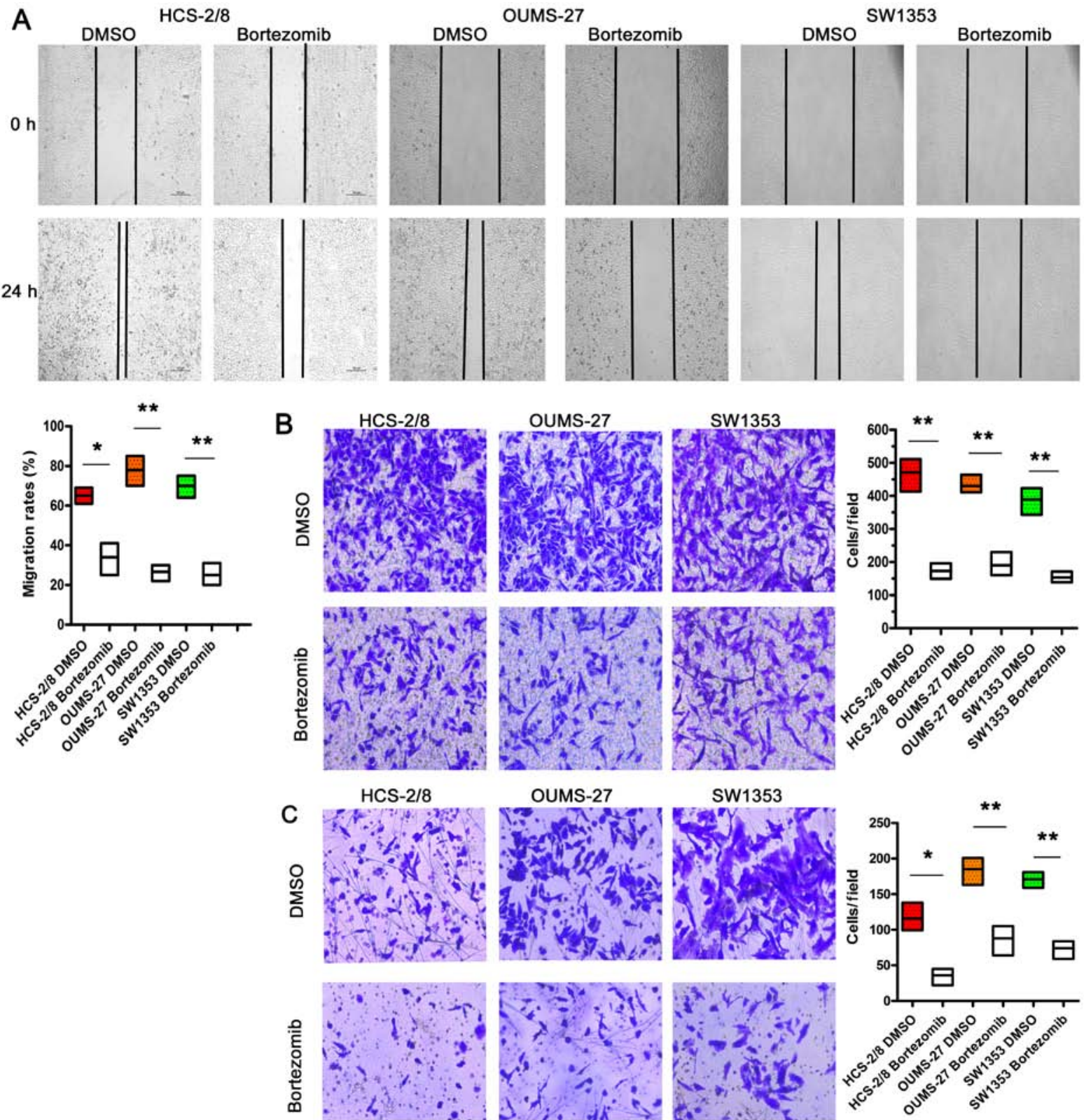

Figure 4. Bortezomib attenuates the migratory and invasive capacities of chondrosarcoma cells. (A) Bortezomib reduces migration of chondrosarcoma cells in wound-healing assays. DMSO or bortezomib were added into the medium of HCS-2/8, OUMS-27 and SW1353 cells after creating the artificial wound. Migration rates were calculated by healing area/wound area $24 \mathrm{~h}$ later. (B) Bortezomib significantly suppresses the migration of cancer cell lines with Transwell analysis. (C) Bortezomib significantly inhibits the invasion of cancer cell lines with Boyden chamber analysis. ( $\left(\mathrm{P}<0.05,{ }^{* *} \mathrm{P}<0.01\right)$.

the effects of bortezomib on cell proliferation and cell cycle arrest by performing cell cycle analysis. Our analysis demonstrated that chondrosarcoma cells were mostly arrested within the G0/G1 phase, indicating there was a reduced number of dividing tumor cells following bortezomib treatment. There were also fewer cells under $\mathrm{S}$ phase, demonstrating that bortezomib could also inhibit DNA replication (Fig. 3B). In addition, cyclin D1, a G0/G1 phase-related protein, was decreased following treatment, as analyzed by western blotting assay (Fig. 3C). Together, these results illustrate that apoptosis, as well as cell G0/G1 phase arrest, are involved in the response of chondrosarcoma to bortezomib treatment.

Bortezomib attenuates the migratory and invasive capacities of chondrosarcoma cells. Chondrosarcoma cells display high migration and invasion capacities, that can be attenuated by bortezomib, as determined by wound healing assay (Fig. 4A) and transwell assays (Fig. 4B), respectively. In the woundhealing assay, bortezomib could very effectively suppress the migration of chondrosarcoma cells. Moreover, treatment with bortezomib resulted in markedly decreased invasive abilities of HCS-2/8, OUMS-27 and SW1353 cells in Matrigel (Fig. 4C). These data show that, in human chondrosarcoma cells, bortezomib effectively attenuates the migratory and invasive capacities.

Bortezomib induces the mesenchymal-epithelial transition of chondrosarcoma cells. Epithelial-mesenchymal transition (EMT) is a critical process for epithelial cells to harbor mesenchymal properties and is closely involved in cancer 

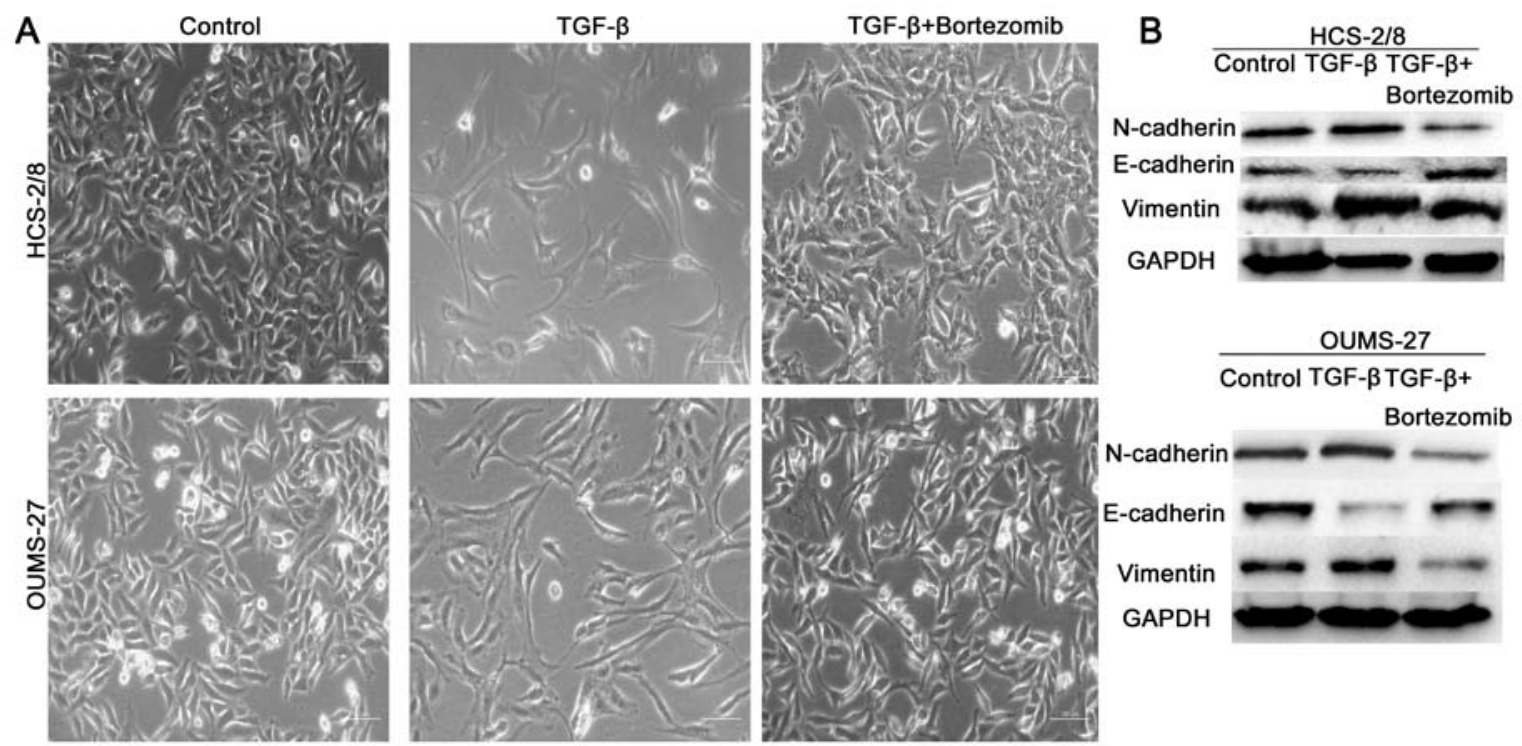

Figure 5. Bortezomib induces the mesenchymal-epithelial transition of chondrosarcoma cells. (A) Bortezomib suppressed the epithelial-related phenotypic traits induced by TGF- $\beta 1$ in chondrosarcoma cells. (B) Protein expression levels of vimentin, N-cadherin and E-cadherin were carried out in HCS-2/8 and OUMS-27 cells with TGF- $\beta 1$ and bortezomib treatment.
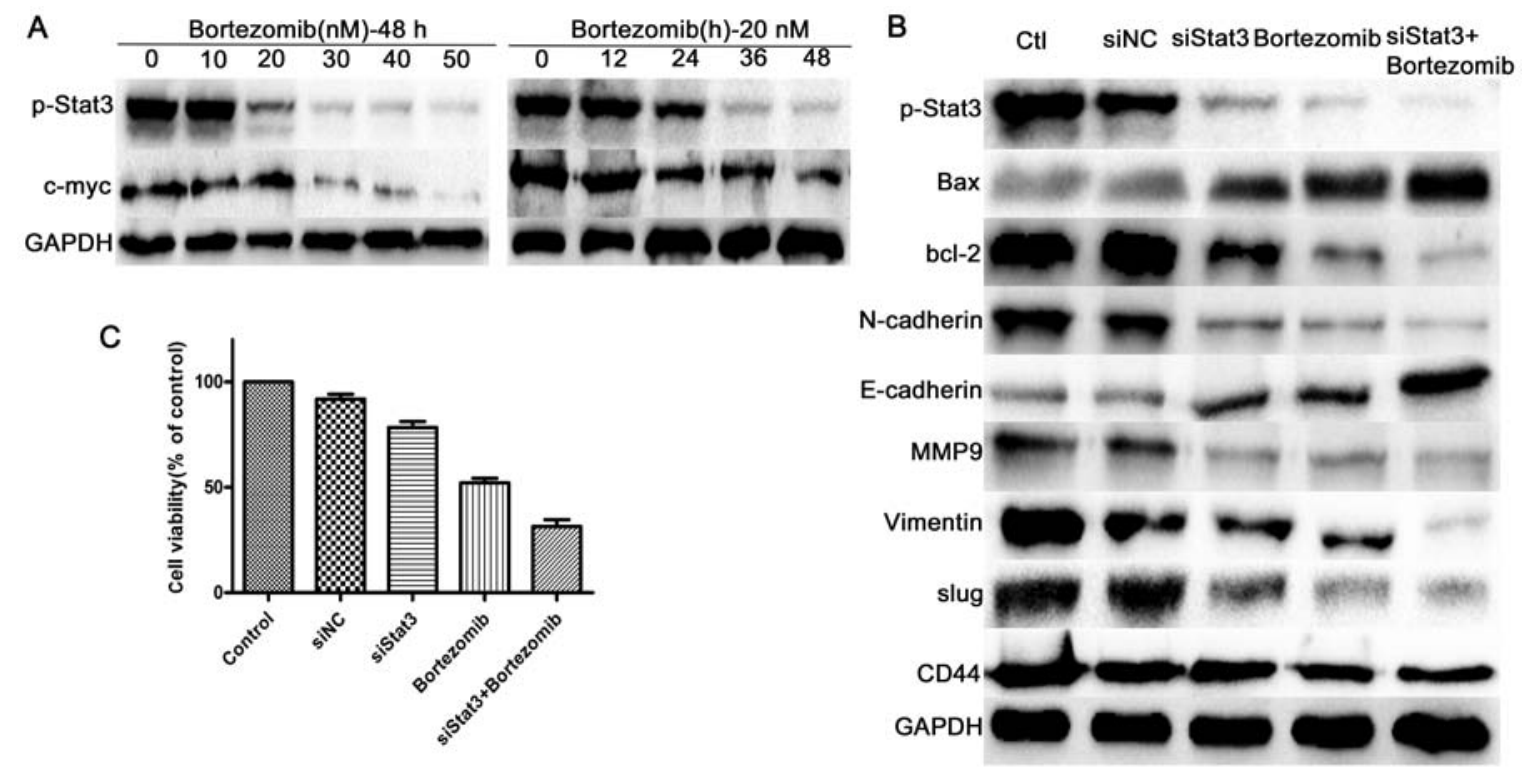

Figure 6. Inactivation of Stat3 signaling contributes to bortezomib-induced effects. (A) Bortezomib treatment repressed expression of p-Stat3 and c-Myc, as assayed by western blot analysis. (B) Western blotting was used to assess p-Stat3, Stat3 targets, apoptosis-related proteins, and EMT-related proteins in Stat3 siRNA- or control siRNA-transfected SW1353 cells treated with bortezomib. (C) The cell viability of siStat 3 silenced SW1353 cells treated with $20 \mathrm{nM}$ bortezomib for $48 \mathrm{~h}$ was decreased more sharply than that treated with siStat 3 or $20 \mathrm{nM}$ bortezomib only, assayed by CCK-8.

invasion and metastasis (27). To evaluate the effect of bortezomib on this process, we enhanced mesenchymal properties of chondrosarcoma cells with TGF- $\beta 1(27,28)$ and found that the cell lines showed mesenchymal appearance, which was fibroblast-like. When treated with bortezomib, however, the chondrosarcoma cell lines maintained an epithelial appearance (Fig. 5A), indicating that bortezomib efficiently induced the mesenchymal-epithelial transition. Moreover, bortezomib impaired the expression of mesenchymal cell markers, including $\mathrm{N}$-cadherin, vimentin and slug. However, the expression of E-cadherin, an epithelial cell marker, increased (Fig. 5B).
Bortezomib inhibits the Stat 3 signalling pathway. As Stat3 is a well-known cancer therapeutic target, we studied whether Stat3 is engaged in bortezomib action in chondrosarcoma cells. Western blotting assay demonstrated that expression of p-Stat3 and its target c-Myc were decreased in SW1353 cells in dose- and time-dependent manner following bortezomib treatment (Fig. 6A). As shown in Fig. 3C, the other two direct downstream targets of Stat3, cyclin D1 and Bcl-2 decreased in the same way.

To further confirm the regulatory role of Stat 3 signaling in bortezomib-treated chondrosarcoma, we characterized the effects of bortezomib in SW1353 cells in which Stat3 


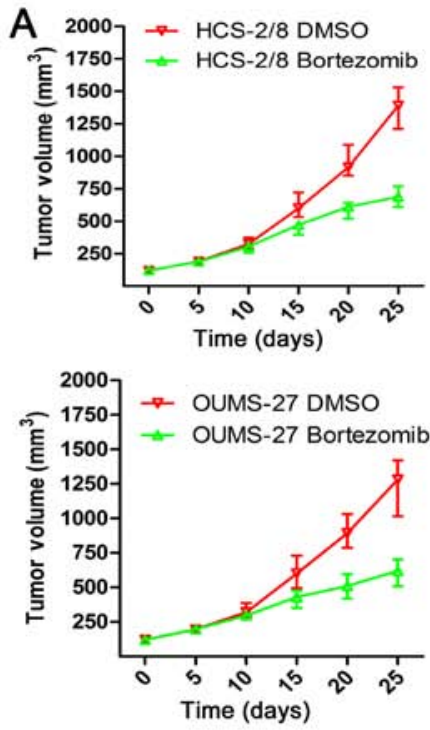

B
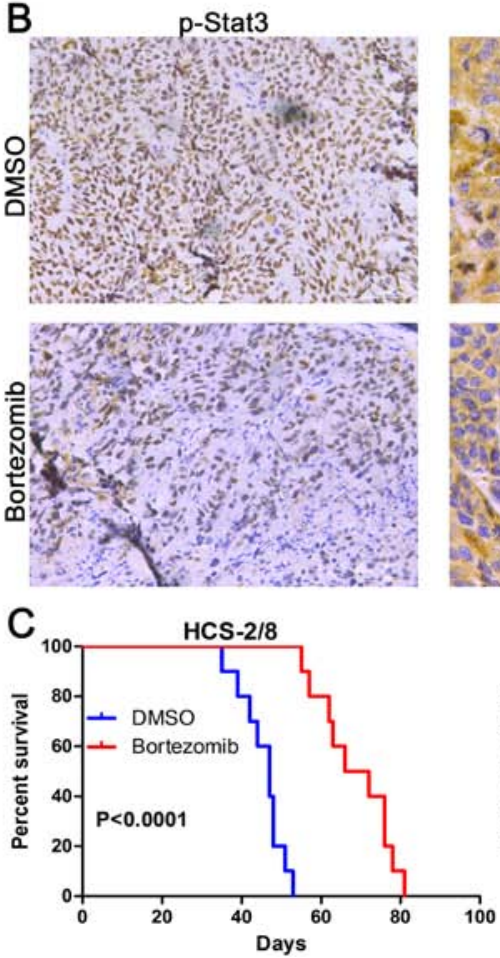

DMSO

Bortezomib

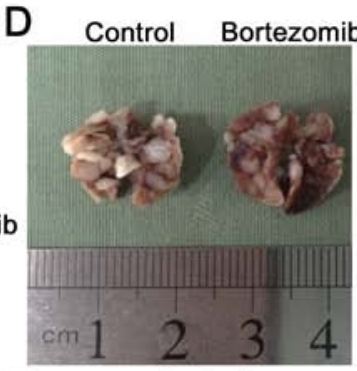

DMSO
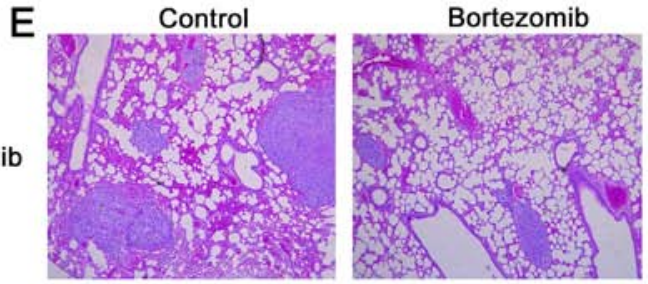

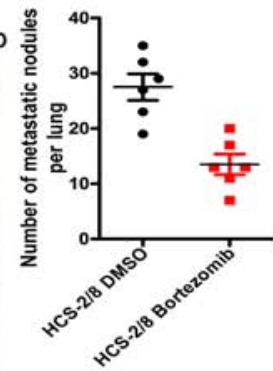

Bortezomib
Bortezomib
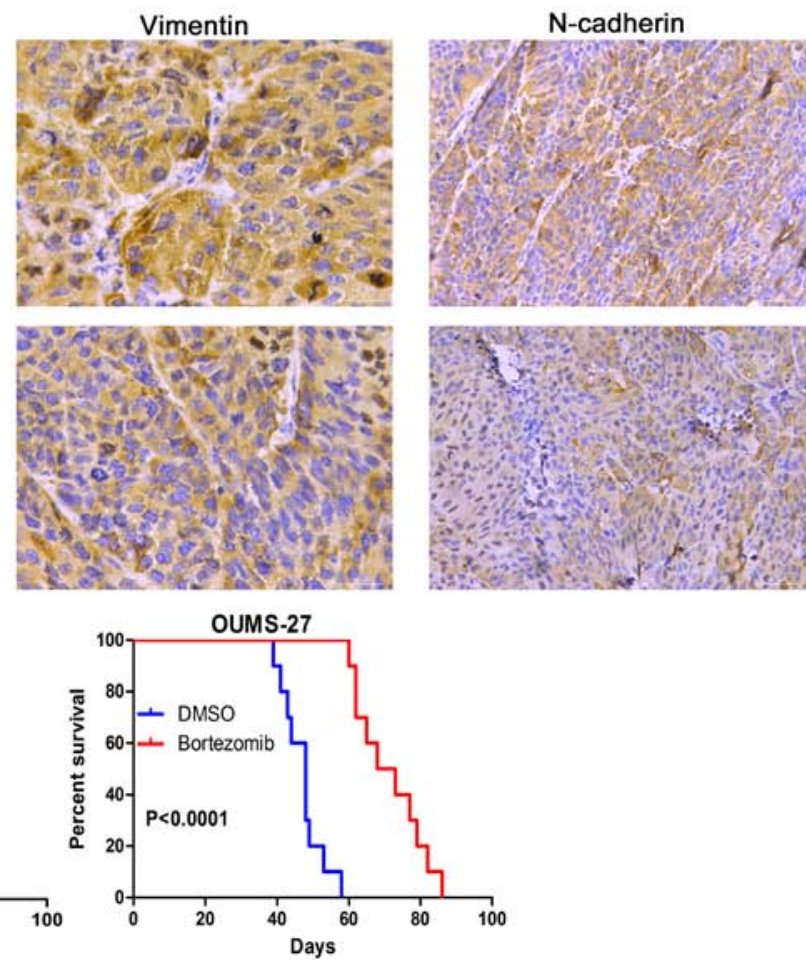

Figure 7. Effects of bortezomib on growth and invasion of chondrosarcoma cells in vivo. (A) The volume of xenografts was measured every five days. The tumors were removed after the mice were sacrificed 30 days later. Data are expressed as mean $\pm \mathrm{SD}$. ${ }^{*} \mathrm{P}<0.05$. (B) Representative images of IHC analysis of p-Stat3, vimentin and N-cadherin in tumors. (C) Bortezomib augments the survival of xenograft mice transplanted with HCS-2/8 and OUMS-27 cells. (D and E) The number of metastatic nodules on the surface of the lungs of mice injected with DMSO or bortezomib is presented. Representative images and H\&E staining of lungs on day 70 after mice were injected with HCS-2/8 cells ( $\mathrm{n}=6$ per group).

was silenced with siRNA. In accordance with bortezomib treatment, knockdown of Stat3 not only induced apoptosis, cell cycle arrest as indicated by increased bax and decreased cyclin D1, but also led to promotion of MET, as evidenced by enhanced E-cadherin expression and decreased N-cadherin, vimentin, MMP-9, CD44 and slug. The expression of p-Stat3 was decreased following siStat 3 or bortezomib treatment for $48 \mathrm{~h}$, while the level of p-Stat 3 was more significantly repressed when treated both siStat 3 and bortezomib, analyzed by western blot assay (Fig. 6B).

Moreover, silencing of Stat 3 resulted in enhanced bortezomib-induced decreases in cell viability. As shown in
Fig. 6C, the cell viability of siStat 3 silenced SW1353 cells treated with $20 \mathrm{nM}$ bortezomib for $48 \mathrm{~h}$ was decreased more significantly than that treated with siStat 3 or only with $20 \mathrm{nM}$ bortezomib. Thus, these data suggest that Stat 3 inactivation is involved in botezomib-mediated inhibition of growth, cell cycle pregression, metastasis and induced apoptosis.

Bortezomib inhibits chondrosarcoma tumor growth in vivo. Five days after HCS-2/8 and OUMS-27 cells were injected subcutaneously into the right armpit of nude BALB/c mice, the mice were randomly divided into two groups and intraperitoneally administered DMSO or botezomib at a dose of $0.5 \mathrm{mg} / \mathrm{kg}$ 
every other day for 30 days. The volume of xenografts was measured every five days (tumor volume $=\left(\right.$ length $\mathrm{x}$ width $\left.{ }^{2}\right) / 2$ ). In line with the in vitro data, bortezomib administration was very effective in inhibiting tumor growth in vivo throughout the course of treatment, resulting in decreased tumor size (Fig. 7A). These data indicate that bortezomib reduces tumor volume and growth rate of chondrosarcoma cells in vivo.

The tumors were removed and evaluated by immunohistochemistry. Bortezomib decreased p-Stat3, vimentin and $\mathrm{N}$-cadherin expression in tumors formed by HCS-2/8 and OUMS-27 cells (Fig. 7B).

Furthermore, bortezomib-treated mice had a significantly longer survival time compared with control mice (Fig. 7C).

Bortezomib inhibits metastasis in vivo. To extend our in vitro observations, we investigated whether bortezomib could regulate tumor metastasis in vivo. After $\mathrm{HCS}-2 / 8$ cells were injected intravenously into the tail vein at 4-weeks, the mice were intraperitoneally administered DMSO or bortezomib at a dose of $0.5 \mathrm{mg} / \mathrm{kg}$ every other day for another 30 days. Bortezomib-treated mice displayed statistically significantly lower numbers of lung metastases than those treated with DMSO (Fig. 7D). Hematoxylin and eosin staining, of the lungs revealed fewer lung metastatic nodes in the mice treated with bortezomib (Fig. 7E).

\section{Discussion}

Chondrosarcoma is the second most frequent type of primary bone cancer following osteosarcoma (1-3), with limited effective treatment options. At present, surgical resection remains the only effective means of treating chondrosarcomas, but remains associated with poor prognosis due to their resistance to adjuvant treatments such as radio- and chemotherapy. However, molecular targeted therapies have completely changed the treatment paradigm of some tumors due to its high efficiency and low toxicity.

Persistent activation of signal transducer and activator of transcription-3 (Stat3) is found in a wide range of solid malignancies of tumors, containing musculoskeletal tumor, such as Ewing's sarcoma and osteosarcoma (13,29-31). In accordance with our studies, we found that the Stat 3 signal pathway is abnormally activated in chondrosarcoma. In addition, inhibiting Stat 3 by specific siRNA could suppress the growth of human chondrosarcoma cells.

Bortezomib is a dipeptidyl boronic acid, which is a selective inhibitor of the proteasome. Many studies have showed that bortezomib can reversibly suppress the proteasome pathway by binding with the $20 \mathrm{~S}$ proteasome complex directly and blocking its enzymatic activity $(17,32)$. However, the effects of bortezomib appear to be cell type-specific or context dependent.

In the present study we found that a low concentration of bortezomib (20 $\mathrm{nM})$ inhibited the growth of chondrosarcoma cells alone and did not affect normal articular chondrocyte cells. Mesenchymal-related traits, which is the key to tumor metastasis, was also efficiently inhibited by bortezomib treatment. Moreover, bortezomib caused cell cycle arrest and apoptosis of chondrosarcoma cells at a low concentration $(20 \mathrm{nM})$. The molecular mechanisms of action of bortezomib included: growth inhibition, apoptosis promotion, cell cycle arrest, promotion of MET and the inactivation of Stat3.

Our findings are consistent with studies which have revealed that bortezomib inhibited tumor growth via inactivation of the Stat3 pathway (33). While some studies have suggested that bortezomib facilitated G0/G1 arrest in cancer cells $(23,34)$, we found that bortezomib markedly induced G0/G1 phase arrest by downregulating cyclin D1, consequently resulting in chondrosarcoma cell quiescent and impeding tumor progression.

EMT is thought to play a significant role in metastasis $(35,36)$. Metastatic relapses are characterized by rapidly proliferating, drug-resistant tumors that are associated with a high mortality rate (37). Metastasis is a complicated process, during which cells undergo phenotypic alteration and migrate away from the primary tumor, survive in the vasculature or lymphatics, and colonize metastatic sites (38). Stat 3 is activated to trigger the molecular events in the EMT of various types of human tumors (39-41).

In the present study, following exposure of chondrosarcoma cells to bortezomib for 6 days, the cells displayed an epithelial-like morphology. Furthermore, there were increased expression of E-cadherin (an epithelial marker) and decreased expressions of $\mathrm{N}$-cadherin and vimentin (mesenchymal markers). In tumors formed by HCS-2/8 and OUMS-27 cells injected into nude BALB/c mice, bortezomib reduced the expression of $\mathrm{N}$-cadherin and vimentin. These results demonstrate that, exposure to bortezomib leads to chondrosarcoma cells undergoing MET in vitro and in vivo.

In conclusion, bortezomib demonstrates an antineoplastic role on chondrosarcoma both in vitro and in vivo. These beneficial effects can be explained by bortezomib-mediated Stat 3 suppression. This study suggests a promising therapeutics target in chondrosarcoma and probably in other kinds of metastatic malignant tumors.

\section{Acknowledgements}

The present study was supported by the National Natural Science Foundation of China (nos. 81572633 and 81472509).

\section{References}

1. O'Neal LW and Ackerman LV: Chondrosarcoma of bone. Cancer 5: 551-577, 1952.

2. Lee FY, Mankin HJ, Fondren G, Gebhardt MC, Springfield DS, Rosenberg AE and Jennings LC: Chondrosarcoma of bone: An assessment of outcome. J Bone Joint Surg Am 81: 326-338, 1999.

3. Bauer HC, Brosjo O, Kreicbergs A and Lindholm J: Low risk of recurrence of enchondroma and low-grade chondrosarcoma in extremities. 80 patients followed for 2-25 years. Acta Orthop Scand 66: 283-288, 1995.

4. Eriksson AI, Schiller A and Mankin HJ: The management of chondrosarcoma of bone. Clin Orthop Relat Res (153): 44-66, 1980.

5. Bovée JV, Cleton-Jansen AM, Taminiau AH and Hogendoorn PC: Emerging pathways in the development of chondrosarcoma of bone and implications for targeted treatment. Lancet Oncol 6: 599-607, 2005

6. Gelderblom H, Hogendoorn PC, Dijkstra SD, van Rijswijk CS Krol AD, Taminiau AH and Bovée JV: The clinical approach towards chondrosarcoma. Oncologist 13: 320-329, 2008.

7. Yu H, Pardoll D and Jove R: STATs in cancer inflammation and immunity: A leading role for STAT3. Nat Rev Cancer 9: 798-809, 2009.

8. Darnell JE Jr: Reflections on STAT3, STAT5, and STAT6 as fat STATs. Proc Natl Acad Sci USA 93: 6221-6224, 1996. 
9. Kijima T, Niwa H, Steinman RA, Drenning SD, Gooding WE, Wentzel AL, Xi S and Grandis JR: STAT3 activation abrogates growth factor dependence and contributes to head and neck squamous cell carcinoma tumor growth in vivo. Cell Growth Differ 13: 355-362, 2002

10. Chen X, Ying Z, Lin X, Lin H, Wu J, Li M and Song L: Acylglycerol kinase augments JAK2/STAT3 signaling in esophageal squamous cells. J Clin Invest 123: 2576-2589, 2013.

11. Bu LL, Deng WW, Huang CF, Liu B, Zhang WF and Sun ZJ: Inhibition of STAT3 reduces proliferation and invasion in salivary gland adenoid cystic carcinoma. Am J Cancer Res 5: 1751-1761, 2015.

12. Feng Y, Ke C, Tang Q, Dong H, Zheng X, Lin W, Ke J, Huang J, Yeung $\mathrm{SC}$ and Zhang $\mathrm{H}$ : Metformin promotes autophagy and apoptosis in esophageal squamous cell carcinoma by downregulating Stat3 signaling. Cell Death Dis 5: e1088, 2014.

13. Anderson JL, Titz B, Akiyama R, Komisopoulou E, Park A, Tap WD, Graeber TG and Denny CT: Phosphoproteomic profiling reveals IL6-mediated paracrine signaling within the Ewing sarcoma family of tumors. Mol Cancer Res 12: 1740-1754, 2014.

14. Wang J, Ni J, Yi S, Song D and Ding M: Protein inhibitor of activated STAT $\mathrm{x} \alpha$ depresses cyclin D and cyclin D kinase, and contributes to the inhibition of osteosarcoma cell progression. Mol Med Rep 13: 1645-1652, 2016.

15. Sandoval-Usme MC, Umaña-Pérez A, Guerra B, HernándezPerera O, García-Castellano JM, Fernández-Pérez L and Sánchez-Gómez M: Simvastatin impairs growth hormoneactivated signal transducer and activator of transcription (STAT) signaling pathway in UMR-106 osteosarcoma cells. PLoS One 9: e87769, 2014.

16. Kane RC, Farrell AT, Sridhara R and Pazdur R: United States Food and Drug Administration approval summary: Bortezomib for the treatment of progressive multiple myeloma after one prior therapy. Clin Cancer Res 12: 2955-2960, 2006.

17. Chen D, Frezza M, Schmitt S, Kanwar J and Dou QP: Bortezomib as the first proteasome inhibitor anticancer drug: Current status and future perspectives. Curr Cancer Drug Targets 11: 239-253, 2011.

18. Cavo M: Proteasome inhibitor bortezomib for the treatment of multiple myeloma. Leukemia 20: 1341-1352, 2006.

19. Shin DH, Chun YS, Lee DS, Huang LE and Park JW: Bortezomib inhibits tumor adaptation to hypoxia by stimulating the FIH-mediated repression of hypoxia-inducible factor-1. Blood 111: 3131-3136, 2008.

20. Mackay H, Hedley D, Major P, Townsley C, Mackenzie M, Vincent M, Degendorfer P, Tsao MS, Nicklee T, Birle D, et al: A phase II trial with pharmacodynamic endpoints of the proteasome inhibitor bortezomib in patients with metastatic colorectal cancer. Clin Cancer Res 11: 5526-5533, 2005.

21. Kim GP, Mahoney MR, Szydlo D, Mok TS, Marshke R, Holen K, Picus J, Boyer M, Pitot HC, Rubin J, et al: An international, multicenter phase II trial of bortezomib in patients with hepatocellular carcinoma. Invest New Drugs 30: 387-394, 2012.

22. Hideshima T, Chauhan D, Hayashi T, Akiyama M, Mitsiades N, Mitsiades C, Podar K, Munshi NC, Richardson PG and Anderson KC: Proteasome inhibitor PS-341 abrogates IL-6 triggered signaling cascades via caspase-dependent downregulation of gp130 in multiple myeloma. Oncogene 22: 8386-8393, 2003.

23. Yang Z, Liu S, Zhu M, Zhang H, Wang J, Xu Q, Lin K, Zhou X, Tao M, Li C and Zhu H: PS341 inhibits hepatocellular and colorectal cancer cells through the FOXO3/CTNNB1 signaling pathway. Sci Rep 6: 22090, 2016

24. Tingting R, Wei G, Changliang P, Xinchang L and Yi Y: Arsenic trioxide inhibits osteosarcoma cell invasiveness via MAPK signaling pathway. Cancer Biol Ther 10: 251-257, 2010.

25. Sun Y, Guo W, Ren T, Liang W, Zhou W, Lu Q, Jiao G and Yan T: Gli1 inhibition suppressed cell growth and cell cycle progression and induced apoptosis as well as autophagy depending on ERK1/2 activity in human chondrosarcoma cells. Cell Death Dis 5: e979, 2014.
26. Sun KX, Xia HW and Xia RL: Anticancer effect of salidroside on colon cancer through inhibiting JAK2/STAT3 signaling pathway. Int J Clin Exp Pathol 8: 615-621, 2015.

27. Lamouille S, Xu J and Derynck R: Molecular mechanisms of epithelial-mesenchymal transition. Nat Rev Mol Cell Biol 15: 178-196, 2014.

28. Xu J, Lamouille S and Derynck R: TGF-beta-induced epithelial to mesenchymal transition. Cell Res 19: 156-172, 2009.

29. Wake MS and Watson CJ: STAT3 the oncogene - still eluding therapy? FEBS J 282: 2600-2611, 2015.

30. Stobbe-Maicherski N, Wolff S, Wolff C, Abel J, Sydlik U, Frauenstein $\mathrm{K}$ and Haarmann-Stemmann T: The interleukin6-type cytokine oncostatin $\mathrm{M}$ induces aryl hydrocarbon receptor expression in a STAT3-dependent manner in human HepG2 hepatoma cells. FEBS J 280: 6681-6690, 2013.

31. Salas S, Jiguet-Jiglaire C, Campion L, Bartoli C, Frassineti F, Deville JL, Maues De Paula A, Forest F, Jézéquel P, Gentet JC, et al: Correlation between ERK1 and STAT3 expression and chemoresistance in patients with conventional osteosarcoma. BMC Cancer 14: 606, 2014

32. Dalla Via L, Nardon C and Fregona D: Targeting the ubiquitinproteasome pathway with inorganic compounds to fight cancer: A challenge for the future. Future Med Chem 4: 525-543, 2012.

33. Lee JH, Kim C, Kim SH, Sethi G and Ahn KS: Farnesol inhibits tumor growth and enhances the anticancer effects of bortezomib in multiple myeloma xenograft mouse model through the modulation of STAT3 signaling pathway. Cancer Lett 360: 280-293, 2015.

34. Kim JE, Lee JI, Jin DH, Lee WJ, Park GB, Kim S, Kim YS, Wu TC, Hur DY and Kim D: Sequential treatment of HPV E6 and E7-expressing TC-1 cells with bortezomib and celecoxib promotes apoptosis through p-p38 MAPK-mediated downregulation of cyclin D1 and CDK2. Oncol Rep 31: 2429-2437, 2014.

35. Liang YJ, Wang QY, Zhou CX, Yin QQ, He M, Yu XT, Cao DX, Chen GQ, He JR and Zhao Q: MiR-124 targets Slug to regulate epithelial-mesenchymal transition and metastasis of breast cancer. Carcinogenesis 34: 713-722, 2013.

36. Micalizzi DS, Farabaugh SM and Ford HL: Epithelialmesenchymal transition in cancer: Parallels between normal development and tumor progression. J Mammary Gland Biol Neoplasia 15: 117-134, 2010.

37. Romero-Pérez L, López-García MÁ, Díaz-Martín J, Biscuola M, Castilla MÁ, Tafe LJ, Garg K, Oliva E, Matias-Guiu X, Soslow RA, et al: ZEB1 overexpression associated with E-cadherin and microRNA-200 downregulation is characteristic of undifferentiated endometrial carcinoma. Mod Pathol 26: 1514-1524, 2013.

38. Lim YY, Wright JA, Attema JL, Gregory PA, Bert AG, Smith E, Thomas D, Lopez AF, Drew PA, Khew-Goodall Y, et al: Epigenetic modulation of the miR-200 family is associated with transition to a breast cancer stem-cell-like state. J Cell Sci 126 2256-2266, 2013.

39. Liu RY, Zeng Y, Lei Z, Wang L, Yang H, Liu Z, Zhao J and Zhang HT: JAK/STAT3 signaling is required for TGF- $\beta$-induced epithelial-mesenchymal transition in lung cancer cells. Int $\mathrm{J}$ Oncol 44: 1643-1651, 2014.

40. Yuan W, Li T, Mo X, Wang X, Liu B, Wang W, Su Y, Xu L and Han W: Knockdown of CMTM3 promotes metastasis of gastric cancer via the STAT3/Twist1/EMT signaling pathway. Oncotarget 7: 29507-29519, 2016.

41. Tania M, Khan MA and Fu J: Epithelial to mesenchymal transition inducing transcription factors and metastatic cancer. Tumour Biol 35: 7335-7342, 2014. 